\title{
On the perturbation of self-organized urban street networks
}

\author{
Jérôme G. M. Benoit ${ }^{\text {* }}$ (1) and Saif Eddin G. Jabari ${ }^{1,2}$
}

\author{
${ }^{*}$ Correspondence: \\ jerome.benoit@nyu.edu \\ ${ }^{1}$ New York University Abu Dhabi, \\ Saadiyat Island, POB 129188, \\ Abu Dhabi, UAE \\ Full list of author information is \\ available at the end of the article
}

\begin{abstract}
We investigate urban street networks as a whole within the frameworks of information physics and statistical physics. Urban street networks are envisaged as evolving social systems subject to a Boltzmann-mesoscopic entropy conservation. For self-organized urban street networks, our paradigm has already allowed us to recover the effectively observed scale-free distribution of roads and to foresee the distribution of junctions. The entropy conservation is interpreted as the conservation of the surprisal of the city-dwellers for their urban street network. In view to extend our investigations to other urban street networks, we consider to perturb our model for self-organized urban street networks by adding an external surprisal drift. We obtain the statistics for slightly drifted self-organized urban street networks. Besides being practical and manageable, this statistics separates the macroscopic evolution scale parameter from the mesoscopic social parameters. This opens the door to observational investigations on the universality of the evolution scale parameter. Ultimately, we argue that the strength of the external surprisal drift might be an indicator for the disengagement of the city-dwellers for their city.

Keywords: Urban street networks, Self-organizing networks, Entropic equilibrium, MaxEnt, Power law, City science, Interdisciplinary physics, Information physics,

Statistical physics, Surprisal, Wholeness, Big data
\end{abstract}

\section{Introduction}

We seek to understand the statistics of urban street networks. Such an understanding will help urban designers and decision makers to improve urban policies in general and urban transportation in particular. In our work we investigate urban street networks as a whole within the frameworks of information physics (Knuth 2011) and statistical physics (Jaynes 1957a, b).

Although the number of times that a natural road crosses an other one has been widely observed to follow a discrete Pareto probability distribution (Clauset et al. 2009) among self-organized cities (Alexander 1965; Crucitti et al. 2006; Jiang et al. 2014), very few efforts have focused on deriving the statistics of urban street networks from fundamental principles. Here a natural road (or road) denotes an accepted substitute for a "named" street (Jiang et al. 2014). In a recent work (Benoit and Jabari 2019), we introduce a statistical physics model that derives the statistics of self-organized urban street networks by applying Jaynes's maximum entropy principle (Jaynes 1957a, b) through the information physics paradigm (Knuth 2011).

(c) The Author(s). 2019 Open Access This article is distributed under the terms of the Creative Commons Attribution 4.0 International License (http://creativecommons.org/licenses/by/4.0/), which permits unrestricted use, distribution, and reproduction in any medium, provided you give appropriate credit to the original author(s) and the source, provide a link to the Creative Commons license, and indicate if changes were made. 
Our approach explicitly emphasizes the road-junction hierarchy of the initial urban street network rather than implicitly splitting it accordingly in two dual but distinct networks. Most of the investigations indeed seek to cast the initial urban street network into a road-road topological network (Jiang et al. 2014) and to describe its valence probability distribution. This holistic viewpoint adopted by the urban community (Alexander 1965; Atkin 1974) appears to fit well with the mindset of information physics (Knuth 2011), which is built upon partial order relations (Davey and Priestley 2002; Knuth 2011). Here the partial order relation derives from the road-junction incidence relation. The passage from the road-junction hierarchy to a Paretian coherence occurs by imposing a Boltzmann-mesoscopic entropy conservation (Milaković 2001; Dover 2004). The emerging statistical physics expresses better in terms of surprisal (Tribus 1961). Surprisal quantifies our astonishment and indecision whenever we face an arbitrary event. Here surprisal betrays the perception of the city-dwellers for their own urban street network. Then, the passage to Paretian coherence simply expresses the conservation on average of their perception-surprisal. Ultimately, we are facing a Paretian statistical physics that challenge our Gaussian way of thinking.

The present work explores, by hand, how we can extend our Paretian statistical physics model for self-organized urban street network to 'nearly' self-organized urban street networks. Basically, we want to proceed by applying arbitrary small perturbations to our model, and see what we get. In the remaining, the paper is organized as follows. The second section articulates the pace from raw urban street networks to idealized selforganized urban street networks within the framework of information physics. Next, the third section shifts to Jaynes's maximum entropy principle. There, along treatments and discussions, we set the idealized self-organized Shannon-Lagrangian for urban street networks before we perturb it with an external surprisal drift. Eventually, after highlighting the two major practical properties of our theoretical work, we point to future observational works around the universality of self-organized urban street networks as such and as reference.

\section{From apparent dullness to living coherence}

\section{Structure to quantify}

From street-junction networks to road-road networks

Everyone has seen black-and-white city maps drew with lines of the same width as shown in Fig. 1g. Each line intersection represents a street-junction (or junction), each portion of line between two adjacent junctions may be identified as a street-segment. Basically, an urban street network is composed of junctions bonded by street-segments. That is, junctions and street-segments constitute, respectively, the immediate nodes and links of a family of real-world networks known as urban street networks - see Fig. 2. As such, these real-world networks are literally street-junction networks. Construction rules readily impose that each junction ties together at least three street-segments. On the other hand, everyday observations tell us that, anywhere in any city, any junction joins mostly four street-segments, occasionally five or six, rarely seven, and very exceptionally more. Real data analysis shows that the valence distribution for street-junction networks essentially follows a Poisson law sharply centred in four (Jiang et al. 2008; Jiang and Liu 2009). In this sense, the complexity of street-junction networks tends to be as trivial as a regular square lattice. This first attempt to describe urban street environments - better known 


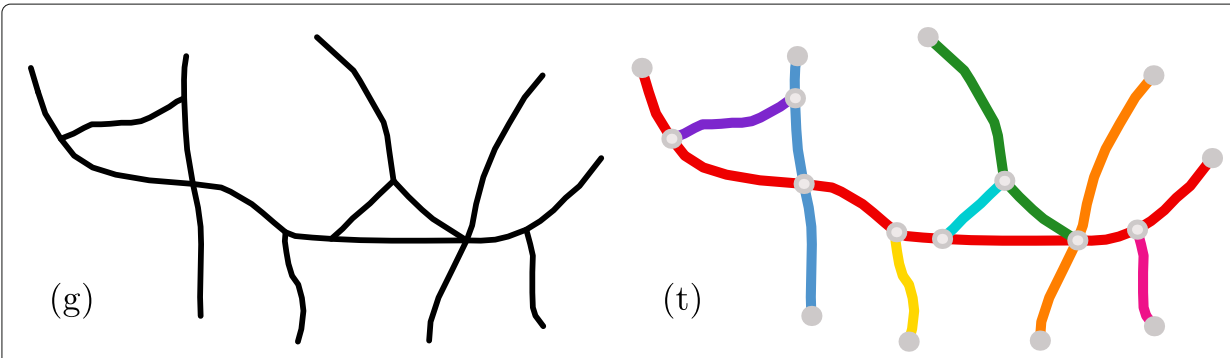

Fig. 1 Notional urban street network ${ }^{1}$ in black-and-white and colourized versions used all along the paper. This notional example is meant to pattern a portion of a real-world city map. The black-and-white version (g) connotes a geometrical viewpoint that leads to a Poissonian physics. Whereas the colourized version ( $t$ ) evinces a topological perception that is subject to scale-free behaviours

as the geometrical approach - may appear to be too naive (Jiang and Claramunt 2004; Jiang and Liu 2009; Jiang et al. 2008, 2014; Masucci et al. 2009; Porta et al. 2006b; Rosvall et al. 2005).

As an alternative, we may consider instead colourized city maps with lines of arbitrary colours as shown in Fig. 1t. We have in mind street maps. Basically, a street map of a city has the particularity to exhibit how the city-dwellers perceive the urban street network of their own city. Explicitly, it shows how they have gathered along the time the streetsegments of their own city to form streets. Implicitly, it reveals that we human townmen rather reason in terms of streets than of street-segments. But over all, deeply, it betrays a topological mindset that looks on street maps essentially for topological information. Indeed, to move from one place to another, we seek for directional information with the following three characteristic traits: (i) each pair of successive streets must critically share a common junction - whichever it is; (ii) each junction in itself plays a secondary role; (iii) neither position nor distance is important. The topological approach forces these three characteristic traits by reducing road maps to (topological) road-road networks. Here a natural road (or road, for short) is an accepted substitute for street (more precisely, for "named" street). A road-road network reduces roads to nodes and bonds each pair that shares a common junction - see Fig. 2. Real data analysis shows that the valence distribution for the road-road network of a self-organized urban street network typically follows an inverse-power scaling law, namely, a scale-free power law (Crucitti et al. 2006; Jiang 2007; Jiang and Claramunt 2004; Jiang et al. 2008, 2014; Porta et al. 2006a, b). This is scalefreeness. We have a slight grasp of scale-freeness for an urban street network whenever we apprehend that only a few streets cross a large number of them, several streets cross an intermediate number of them, and very many streets cross a small number of them. As a matter of fact, by contrast to street-junction networks, road-road networks are subject to complex network behaviours.

Thusly, the topological approach appears far more pertinent than the geometrical one for at least two reasons. Firstly, the topological description unveils that urban street networks underlie complex behaviours generally observed in real complex networks (Jiang et al. 2008, 2014; Jiang and Liu 2009; Masucci et al. 2009; Masucci and Molinero 2016; Porta et al. 2006b; Rosvall et al. 2005); the complexity induced by the geometrical description is trivial (Jiang et al. 2008; Jiang and Liu 2009). Secondly, the topological approach permits to isolate a category of real urban street networks that shows evidence of a 'pure' scaling behaviour; the geometrical approach renders all urban street networks equally 


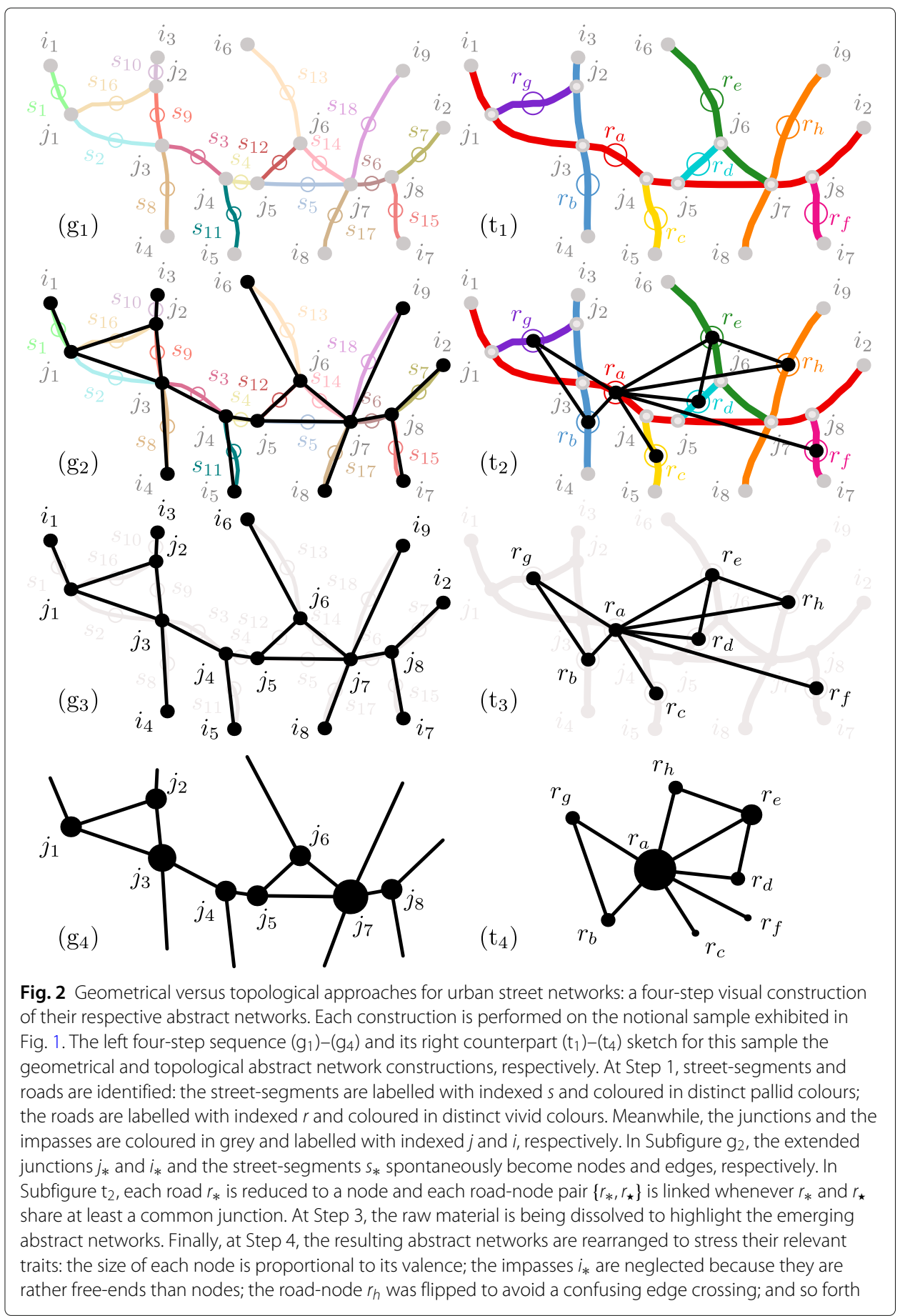

'boring' (Jiang et al. 2008). This idealized category of urban street networks may serve as a reference from which any general urban street networks deviates.

\section{Road-road networks mask road-junction partial orders}

Even though the topological approach leads to precious observations, it remains mostly a descriptive tool. The topological approach does not provide any explanation, it is not concerned about the underlying principles for how urban street networks are emerging. 
A structural approach that does not bypass street-junctions (or road-junctions) allows us to establish a statistical physics foundation for the 'pure' scaling behaviour as effectively observed among self-organized urban street networks (Benoit and Jabari 2019). It is fair to add that the structural approach may lead to alternative foundations, but also that it does not fail to catch the 'true structure' of urban street networks by forcing the three above topological characteristic traits a bit too early.

Here urban street networks are envisioned as a whole where road literally tie together through junctions. To begin with, we represent the ties by an incidence relation that gathers for each road all junctions through which it passes (Jiang et al. 2008) as exemplified in Table 1. Then, we interpret this road-junction incidence relation as an object/attribute relation for which any road acts as an objects and any junction as an attribute (Atkin 1974; Davey and Priestley 2002; Ho 1982). Eventually, by invoking the Formal Concept Analysis (FCA) paradigm, this change of perspective allows us to establish bijectively a partialorder relation (Davey and Priestley 2002; Ho 1982). In other words, every urban street network is subject to and bijectively representable by a partial-order.

More interestingly, any partial-order can be represented by an abstract ordered structure known as Galois lattice (Davey and Priestley 2002; Ho 1982). In general, a Galois lattice organizes itself in layers with respect to its partial-order, so that it can give rise to sympathetic graphical representations called Hasse diagrams (Davey and Priestley 2002). The Galois lattice corresponding to the incidence relation in Table 1 is represented by a Hasse diagram in Fig. 3. If we assume that two roads cross to each other only once, it appears then that urban street networks reduce to intuitive two-layer Galois lattices: the roads and the junctions make up the lower nontrivial layer and the upper nontrivial layer, respectively; the 'imply' ordering relation (or join operator) is "passing through" (or "crossing at"). Figure 3 exhibits clearly this property. Roads that cross to each other more than once form loops. Since such loops are rare while mostly not spontaneous, for the sake of simplicity and unless otherwise specify, the remaining will consider set of roads free of such loops.

Distributivity is an important property of Galois lattices (Davey and Priestley 2002). In particular, for any finite Galois lattice, distributivity allows us to claim that the elements of the first nontrivial layer are the join-irreducible elements of the Galois lattice; that is, each upper elements can be expressed as a join chain composed with elements of the first nontrivial layer - while none element of the first nontrivial layer can be decomposed. In our

Table 1 Road-junction incidence dot-chart associated to the colourized notional urban street network introduced in Fig. 1 with the labelling chosen in Fig. 2

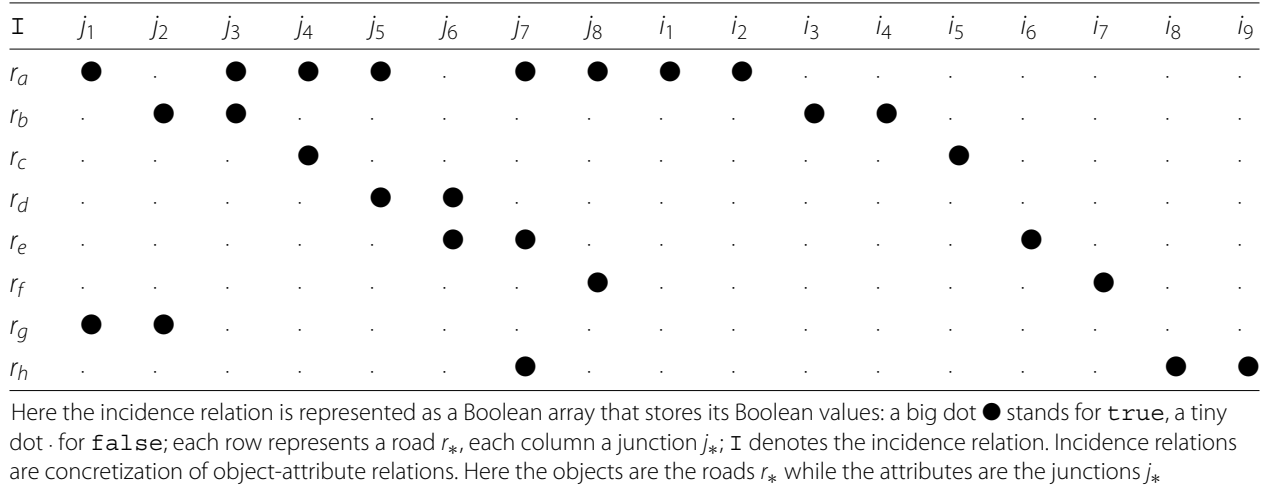




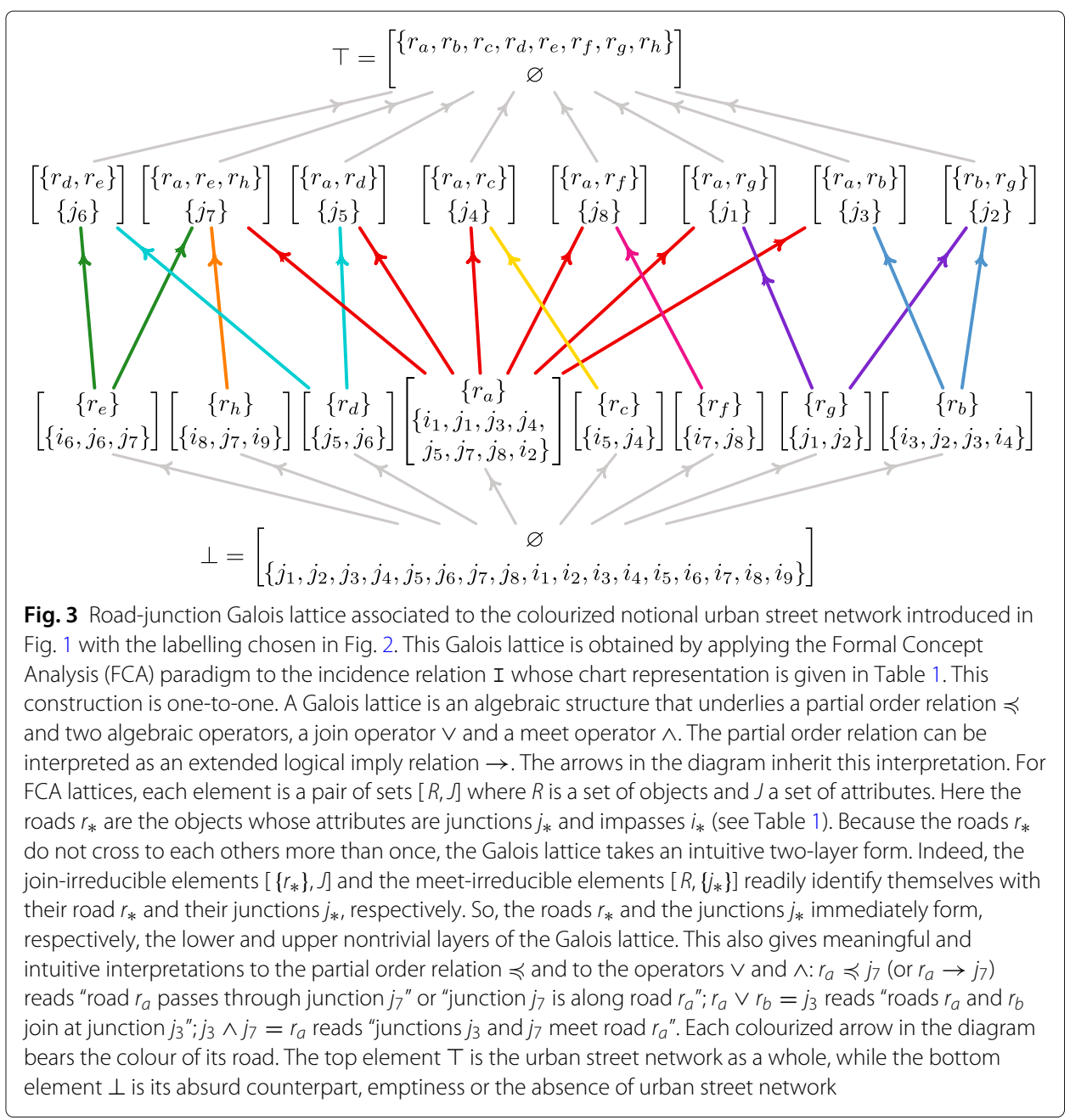

context, distributivity corresponds to the intuition that any junction is a crossing of only two roads. Therefore, any junction that joins more than two roads render the underlying Galois lattice nondistributive. However, as we have seen above, junctions mostly join two roads. Second, any junction that joins at least three roads can be replaced by a roundabout so that it remains only junctions that joins at most two roads. For theses two reasons, we may qualify as canonical any urban street network whose junctions effectively join only two roads.

Furthermore, it is noticeable that the underlying Galois lattice proves not only to reduce bijectively but also to reflect pertinently the involving topological complexity. Indeed, each underlying Galois lattice assigns a clear primary role to roads and a clear secondary role to junctions so that the three topological characteristic traits are valorized as they should be: roads imply junctions; roads are join-irreducible (or just irreducible, for short), while junctions are join-reducible (reducible) to roads; junctions are meetirreducible, while roads are meet-reducible to junctions. [ As an aside, for roads that form loops with each others, the FCA paradigm simply creates abstractions of roads and junctions: roads and junctions may then be defined, respectively, as the join-irreducible and meet-irreducible elements of the involved road-junction Galois lattice. ] By now, most of 
us should recognize road-road networks as either zeroth order approximations or projections of road-junction Galois lattices by employing either an analytic analogy or a geometric one, respectively.

To summarize, any urban street network bijectively reduces its topological complexity to an essentially distributive two-layer Galois lattice, while its canonicalization renders the latter plainly distributive.

\section{Partial orders are algebraic structures}

Actually, Galois lattices are not only ordered structures but also algebraic structures (Davey and Priestley 2002; Ho 1982). To put it another way, the join operator (or partial order) not only permit us to construct the entire Galois lattice from its join-irreducible elements (Davey and Priestley 2002; Ho 1982) but also to consistently assign numbers to its elements so that the algebra of these numbers reflects the algebra of the Galois lattice while their order respects its partial-order (Knuth 2005, 2008, 2009, 2011, 2014). The evaluation of partially ordered sets (or Galois lattices) is the main object of the theory of information physics (Knuth 2005, 2008, 2009, 2011, 2014). Because quantifying roads and their concomitant junctions enable us to confront models motivated by principles against observed data, the structural approach liberates us to restrain ourselves to perform sophisticated but nevertheless blind data analysis. Given this, the structural approach may no more appear as a gratuitous æsthetic step to the most skeptical readers. In brief, the structural approach is a game changer.

In fact, three Galois lattices are getting involved (Knuth 2008, 2009). Let us now, in order to forge for ourselves a better comprehensive picture, succinctly describe them and their respective valuation functions. The task is relatively easy since we are already familiar with the Galois lattice of our system, with the valuation function of the first extra Galois lattice, and almost with the valuation function of the last Galois lattice. Of course, our first and foremost Galois lattice is the system itself, so that our unique unknown valuation function Va is simply meant to describe the physics of our system. Specifically, the unknown valuation function $\mathrm{Va}$ assigns a positive real number indiscriminately to all roads and junctions so that each assigned positive real number characterises the physical state of the involved road or junction. Notice that valuation functions must be positive for consistency reasons. As generic Galois lattice components, roads and junctions organizes themselves in downsets. A downset is a set of elements which contains all the elements implying each of them (Davey and Priestley 2002). If we mentally sketch our urban street network randomly by roads and junctions under the unique rule that a junction can be dotted only when all of its joining roads are already lined, then each downset represents a state of our mental picture - and vice versa. The set of all downsets ordered according to set inclusion $\subseteq$ forms a distributive Galois lattice, which is called the state space. The state space is an auxiliary Galois lattice which merely helps us to introduce the next relevant one.

The join-irreducibles of the state space are the downsets associated to every road or junction, that is, the singleton sets composed of one road and sets composed of one junction along all of its joining roads. These join-irreducibles generate the state space with set union $\cup$ as join-operator. Nevertheless, in reality, given city-dwellers may not know precisely which state their mental picture of the urban street network represents. Even so, they may have some information that exclude some states, but not others. Therefore, the 
mental pictures of city-dwellers are mostly sets of potential states than single states know with certainty. A set of potential states is called a statement. The set of all possible statements is simply the powerset generated from the set of all states. Once ordered according to set inclusion $\subseteq$, the set of all statements becomes a distributive Galois lattice whose the join-irreducibles are the states. This Galois lattice is known as the hypothesis space. Within the hypothesis space, statements follows a logical deduction order as each statement literally implies (or is included in) a statement with certainty. The valuation function associated to any hypothesis space is recognized to be a probability distribution. So, we are already very familiar with the algebra satisfied by the valuation functions associated to hypothesis spaces. Among valuation functions associated to Galois lattices, this algebra can be shown to be the only one possible by imposing natural algebraic consistency restrictions.

Let us digress briefly to bring our attention back to the system valuation function Va: as immediate consequence, for canonical urban street networks, the evaluation $\mathrm{Va}(j(r, s))$ of a junction $j(r, s)$ joining a pair of roads $(r, s)$ must be the sum of the evaluations $\mathrm{Va}(r)$ and $\mathrm{Va}(s)$ of the joined roads $r$ and $s$, respectively; we have

$$
\mathrm{Va}(j(r, s))=\mathrm{Va}(r)+\mathrm{Va}(s) .
$$

End of digression.

Because the hypothesis state is essentially a representation of the system, it is reasonable to claim that its valuation function Pr must be related to the valuation function Va of our system, that is, to the physics of our system. Meanwhile, Rota theorem (Rota (1971), Thm. 1, Cor. 2) asserts that, for a finite distributive Galois lattice, the valuation function is perfectly determined by the arbitrary values taken by its join-irreducibles. In other words, the valuation function Pr does not depend on the very structure of the hypothesis space; rather, it depends on the arbitrary values assigned to the join-irreducibles of the hypothesis space, which are the states. Accordingly, the probability assigned to each state has to be an arbitrary function of its evaluation by the valuation function Va; this is a composition. This arbitrary function interprets itself as a weight function $w$. We read

$$
\operatorname{Pr}=w \circ \mathrm{Va} .
$$

The weight function $w$ constitutes our second unknown function. The construction of the hypothesis space from the state space corresponds technically to an exponentiation (Davey and Priestley 2002).

The exponentiation of the hypothesis space brings up an inquiry space. The inquiry space is a distributive Galois lattice whose elements are questions. Thus, by construction, any question is a set of statements that answer it. The quantification of the inquiry space leads to a measure, coined relevance. In fact, the inquiry space is Carrollian in the sense that it contains both vain (and fanciful) and real questions. A respond to a real question is a true state of our system. To wit, a real question permits to know the configuration of our system exactly and without ambiguity. A vain question can only lead to partial or ambiguous knowledge of the configuration. The join chain of all the join-irreducible questions is the smallest real question, it is called the central issue. The questions above the central issue form a Galois sublattice that contains all and only real questions. The joinirreducible elements of the real Galois sublattice appears to partition their answers. This property is reflected in the choice of the relevance by coercing the relevance of a partition 
question to depend on the probability of the greatest statements of its partitions. This choice imposes the relevance to satisfy the four natural properties of entropies (Aczél et al. 1974). This means that relevance is a generalized measure of information with Shannon entropy as basis (Aczél et al. 1974). This is one of the major results of information physics. The relevance of the central issue identifies itself with the entropy. Therefore, for canonical urban street networks, the functional entropy $H[\mathrm{Va}, w]$ takes the form

$$
H[\mathrm{Va}, w]=\sum_{r}(\mathrm{~h} \circ w)(\mathrm{Va}(r))+\sum_{j(r, s)}(\mathrm{h} \circ w)(\mathrm{Va}(r)+\mathrm{Va}(s))
$$

where the first summation runs over the roads $r$ and the second one over the junctions $j(r, s)$ joining the pair of roads $(r, s)$, while $\mathrm{h}: x \mapsto-x \ln x$ is the Shannon entropy function. We will keep to express information measures in nat units. For further details on the theory of information physics, we refer the reader to the work of (Knuth 2005, 2008, 2009, 2011, 2014). For now, we have enough material to step forward.

\section{Quantify to organize}

\section{From Galoisean hierarchy to Paretian coherence}

Network data analysis shows that city-dwellers have a topological perception of their urban street networks. On the other hand, the topology of urban street networks hides a simple road-junction partial order that bijectively reduces to intuitive two-layer Galois lattices. The Galoisean hierarchy is intuitive in the sense that its join-operator expresses our intuition that two roads join to form a junction. Nonetheless this intuitive hierarchy leads to two layers whose cardinality might be perceived as incommensurable. Typical big cities count far more than several roads and junctions. The apparent simplicity of the underlying Galois lattices is the result of an algorithmic thought. Nonetheless the Galoisean hierarchy is three-fold. While the ordering and algebraic perspectives are respectively structural and operational, the whole is measurable. The underlying algebraic structure leads unambiguously to a unique quantification modulo two unknown functions that we are free to choose. These two unknown functions are of different nature. The valuation function $\mathrm{Va}$ assigns to each road or junction of the urban street network a numerical quantity that characterizes its physical state. The weight function $w$, or more precisely its composition with the valuation function $\mathrm{Va}$ as expressed in (2), allows us to assign to each mental picture of the urban street network a numerical quantity that characterizes its perception among the city-dwellers. This assignment is simply the probability distribution Pr of our system. Ultimately all these mental pictures are surrounded by all sort of questions whose pertinence can be measured. The relevance of the most pertinent question is better known as the entropy of the system. The most plausible probability Pr, that is, the quantification which tends to represent at best the perception of the city-dwellers for their own urban street network, must also be the most relevant one. In other words, the most plausible probability Pr must maximize the functional entropy (3) of their urban street network. This is nothing other than Jaynes's maximum entropy principle (Jaynes 1957a, b; 1989; Kapur and Kesavan 1992; Kesavan 2009; Knuth 2008). Thusly, our physical content shifts from a algorithmic order to a fluctuating organization.

Roads and junctions indiscriminately yield our initial ignorance (Jaynes 1989). The most we can tell is that roads and junctions are mesoscopic systems with a finite number of possible configurations $\Omega$. Besides, we must assume our complete ignorance about their 
respective inner worlds. This means that, to our eyes at least, all their possible configurations are equally likely. Thusly, roads and junctions are Boltzmannian mesoscopic systems. Therefore, the probability distribution Pr reduces to a function that depends only on the number of possible configurations $\Omega$. Meanwhile, the functional entropy (3) simplifies to take the more sympathetic form

$$
H[\operatorname{Pr}]=-\sum_{\Omega} \operatorname{Pr}(\Omega) \ln (\operatorname{Pr}(\Omega)) .
$$

On the other hand, here, self-organized urban street networks are idealized as scale-free systems, viz., as systems exhibiting no typical number of configurations but rather a typical scale $\lambda$. Thus, as suitable characterizing moments to invoke Jaynes's maximum entropy principle (Jaynes 1957a, b; Kesavan 2009), we must discard any classical moment and may consider logarithmic moments instead. It appears that imposing the first logarithmic moment

$$
\sum \operatorname{Pr}(\Omega) \ln \Omega
$$

as sole characterizing constraint gives rise to the scale-free probability distribution

$$
\operatorname{Pr}(\Omega) \propto \Omega^{-\lambda}
$$

A practical normalization of this probability distribution leads to the discrete Pareto probability distribution (Clauset et al. 2009). To sum up: the passage from the underlying Galoisean hierarchy to an underlying Paretian coherence occurs by invoking Jaynes's maximum entropy principle with the first logarithmic moment as sole characterizing moment and with our complete ignorance as initial knowledge condition.

For every road or junction having $\Omega$ possible configurations, the Boltzmann entropy $\ln \Omega$ measures nothing but our complete ignorance on the configuration effectively taking place. So, our characterizing restriction simply claims that an idealized self-organized urban street network evolves by preserving our complete ignorance on average. This characterizing scheme that induces a Paretian coherence has been interpreted as some evolutionary based mechanism to maintain some opaque internal order (Milaković 2001; Dover 2004). Note furthermore that "complete ignorance" has rather remained, so far, a technical term. A more intuitive interpretation might be considered instead. If the Boltzmann entropy $\ln \Omega$ is interpreted as the surprisal that city-dwellers associate to every road or junction having $\Omega$ possible configurations, then $\sum \operatorname{Pr}(\Omega) \ln \Omega$ becomes the amount of surprisal on average that they associate to their own urban street network. Surprisal (or surprise) $\mathrm{Su}=-\ln \circ \mathrm{Pr}$ was introduced by Tribus (1961) as a measure to quantify our astonishment and our indecision whenever we face any arbitrary event. Once adapted to our context, surprisal somehow betrays the perception of the city-dwellers for their own urban street network. Therefore, the above Paretian characterizing constraint simply asserts that an idealized self-organized urban street network evolves by preserving on average the perception that its city-dwellers share for it. This assertion renders city-dwellers the unconscious but nevertheless active actors of their own urban street networks, not the passive subjects of an obscure technical machinery. Along this line, the scale parameter $\lambda$ of the underlying scale-free probability distribution (6) interprets itself as an evolution scale. 


\section{Untangling the underlying coherence}

The underlying coherence, Paretian or not, does not reveal to city-dwellers as-is. Technically, we must still untangle the corresponding weight function $w$ and valuation function Va with respect to the underlying algebraic structure, namely, with respect to composition (2) and addition rule (1). Practically, we need a mesoscopic model to count the number of configurations $\Omega$ associated to every road or junction. For the reason that roads and junctions are likely driven by social interactions, the mesoscopic model must typify social interactions. To fulfill this purpose, it appears convenient to adopt and adapt the network of intraconnected agents model introduced by Dover (2004) for the distribution of cities in countries. Thereby, each road or junction becomes a hive of agents that connect to each other. As agents, we may consider the inhabitants that somehow participate to the live activity of roads: drivers, cyclists, pedestrians, suppliers, institutional agents, residents, and so forth. For each road $r$, the number of agents is assumed to be asymptotically proportional to the number of junctions $n_{r}$ that $r$ crosses - the ratio $A$ being constant and sufficiently large. This expresses nothing but the extensive property of roads. Here the very existence of every road relies on the ability for each of its agent to maintain a crucial number of intraconnections which is crudely equal to a constant number $v_{\mathrm{r}}$ (Dover 2004; Dunbar and Shultz 2007), called the number of vital connections for roads. The layout of theses intraconnections is implicitly associated to the internal order within each road, while the total number of possible layouts for each road is simplistically considered as its number of configurations (Dover 2004). Therefore, for each road $r$, the number of configurations $\Omega_{r}$ yields

$$
\Omega_{r}=\Omega_{r}\left(n_{r}\right) \simeq\left(\begin{array}{c}
\frac{1}{2} A n_{r}\left(A n_{r}-1\right) \\
v_{\mathrm{r}}
\end{array}\right) \simeq \frac{A^{2 v_{\mathrm{r}}}}{2^{v_{\mathrm{r}}} v_{\mathrm{r}} !} n_{r}^{2 v_{\mathrm{r}}}
$$

As concerns each junction, continuing along this spirit, the involved agents are merely the agents of the two joining natural roads combined together. Nevertheless, as there is no apparent reason for roads and junctions to experience the same type of internal equilibrium, we will assume two distinct numbers of vital connections, $v_{\mathrm{r}}$ and $v_{\mathrm{j}}$ respectively. Then the same crude maneuvers give

$$
\Omega_{j(r, s)}=\Omega_{j}\left(n_{j}=n_{r}+n_{s}\right) \simeq \frac{A^{2 v_{\mathrm{j}}}}{2^{v_{\mathrm{j}}} v_{\mathrm{j}} !} n_{j}^{2 v_{\mathrm{j}}} .
$$

Therefore, the valuation function Va appears clearly to assign to each road or junction the number of its agents, and the weight function $w$ to asymptotically count the number of possible vital intraconnection layouts - modulo normalization.

\section{Self-organized urban street networks as reference Ideal self-organized urban street networks Coherence based on Boltzmannian mesoscopic surprisals}

It is time now to explicitly invoke Jaynes's maximum entropy principle for the functional entropy (4) with the first logarithmic moment (5) as single characterizing constraint. Promptly, the corresponding Shannon Lagrangian writes 


$$
\begin{aligned}
\mathcal{L}(\{\operatorname{Pr}(\Omega)\} ; \nu, \lambda) & =-\sum_{\Omega} \operatorname{Pr}(\Omega) \ln (\operatorname{Pr}(\Omega))-(v-1)\left[\sum_{\Omega} \operatorname{Pr}(\Omega)-1\right] \\
-\lambda & {\left[\sum_{\Omega} \operatorname{Pr}(\Omega) \ln \Omega-\langle\mathrm{S}\rangle\right] . }
\end{aligned}
$$

The constraint relative to the Lagrange multiplier $\lambda$ compels to keep constant the first logarithmic moment (5) of the probability distribution Pr; namely, it imposes the preservation on average of the amount of surprisal that city-dwellers perceive for their roads and junctions. Meanwhile, the Lagrange multiplier $v$ ensures the normalization condition that the probability distribution Pr must satisfy. The constant $\langle\mathrm{S}\rangle$ stands for the constant mean surprisal at which the system evolves - for now it plays a dummy role. Extremizing expression (8) yields

$$
\frac{\partial \mathcal{L}(\{\operatorname{Pr}(\Omega)\} ; \nu, \lambda)}{\partial \operatorname{Pr}(\Omega)}=-\ln (\operatorname{Pr}(\Omega))-v-\lambda \ln \Omega=0,
$$

which immediately leads to the scale-free probability distribution

$$
\operatorname{Pr}(\Omega)=\frac{\Omega^{-\lambda}}{e^{\nu}}
$$

as previously claimed. Afterwards, the normalization condition effortlessly gives us an expression for the dependent exponential denominator $\exp (v)$, which may be defined as the partition function $Z(\lambda)$ of our system; we have

$$
e^{\nu}=\sum_{\Omega} \Omega^{-\lambda} \equiv Z(\lambda)
$$

Ultimately, we write solution (10) in the more familiar form

$$
\operatorname{Pr}(\Omega)=\frac{\Omega^{-\lambda}}{Z(\lambda)}
$$

The found most plausible probability distribution (12) concerns the underlying coherence of our system. As such, this coherence can only be perceived indirectly by the city-dwellers of the urban street network. The city-dwellers may rather perceive the coherence behind roads and junctions. Their corresponding statistics are obtained as follows. Substituting (7a) into (12), we readily obtain for roads

$$
\operatorname{Pr}\left(n_{r}\right) \propto n_{r}^{-2 \lambda v_{\mathrm{r}}},
$$

which is a scale-free probability distribution. Injecting instead (7b) into (12), then gathering and counting with respect to the precedent probability distribution (13a) gives for junctions

$$
\operatorname{Pr}\left(n_{j}\right) \propto\left(\sum_{j(r, s)} \frac{\left[n_{j}=n_{r}+n_{s}\right]}{\left(n_{r} n_{s}\right)^{2 \lambda v_{\mathrm{r}}}}\right) n_{j}^{-2 \lambda v_{\mathrm{j}}},
$$

which is a generalized power law probability distribution; the summation in parentheses is simply the self-convolution of the road probability distribution (13a). The bracket around the equality statement follows Iverson's convention (Graham et al. 1994; Knuth 1992): the bracket has value one whenever the bracketed statement is true, zero otherwise. The number of junction $n_{r}$ that a road crosses is essentially the number of roads with which it shares a common junction, namely, its valence number in the corresponding road-road network. So the probability distribution (13a) predicts the valence distribution for roads that has been widely observed empirically among self-organized cities (Crucitti et al. 2006; 
Jiang 2007; Jiang and Claramunt 2004; Jiang et al. 2008, 2014; Porta et al. 2006a, b). A similar argument dually applies for junctions. However, to the best of our knowledge, the valence distribution for junctions has brought no attention until now - except in our recent investigations.

For practical data analysis (Clauset et al. 2009), we need to assume that the number of junctions per road $n_{r}$ spans from some minimal positive value $\underline{n}_{r}$. Then the normalization of probability distributions (13) can be performed elegantly by using natural generalization of known special functions. First, the probability for a road to cross $n_{r}$ junctions becomes

$$
\operatorname{Pr}\left(n_{r}\right)=\frac{n_{r}^{-2 \lambda v_{r}}}{\zeta\left(2 \lambda v_{r} ; \underline{n}_{r}\right)},
$$

where $\zeta(\alpha ; a)=\sum_{n=0}^{\infty}(a+n)^{-\alpha}$ is the generalized (or Hurwitz-) zeta function (Olver et al. (2010), $\mathbb{\$} 25.11$ ). Second, the probability for a junction to see $n_{j}$ junctions through its joining roads reads

$$
\operatorname{Pr}\left(n_{j}\right)=\frac{\sum_{n=\underline{n}_{r}}^{n_{j}-\underline{n}_{r}}\left[n\left(n_{j}-n\right)\right]^{-2 \lambda v_{r}} n_{j}^{-2 \lambda v_{j}}}{\mathcal{W}\left(2 \lambda v_{r}, 2 \lambda v_{r}, 2 \lambda v_{j} ; \underline{n}_{r}\right)},
$$

where $\mathcal{W}(\alpha, \beta, \gamma ; \underline{n})=\sum_{m, n \geqslant \underline{n}} m^{-\alpha} n^{-\beta}(m+n)^{-\gamma}$ is the two-dimensional generalized (or Hurwitz-) Mordell-Tornheim-Witten zeta function (Borwein and Dilcher 2018).

As a conclusion, let us remark that statistics (14) for an ideal self-organized urban street network does not separate the macroscopic parameter $\lambda$ from the mesoscopic ones $v_{\mathrm{r}}$ and $v_{\mathrm{j}}$ in the sense that, at best, we can only estimate the products $\lambda v_{\mathrm{r}}$ and $\lambda v_{\mathrm{j}}$. This separation of parameters is critical since it would allow us to distinguish quantitatively the macroscopic phenomenon of evolution from the mesoscopic phenomena of social interactions that take place in urban street networks. Notice that, from a qualitative perspective, two distinct behaviours are anticipated. The numbers of vital connections $v_{\mathrm{r}}$ and $v_{\mathrm{j}}$ certainly differ from one cultural basin to another one (Dover 2004). Whereas the evolution scale $\lambda$ might transcend cultures (West 2017). A classical way to separate parameters in Physics consists to introduce sufficiently small perturbations. This is, in its observational form, the subject of the next subsection.

\section{Case study of Central London}

Figure 4 shows the Relative Frequency Distributions (RFD) of the urban street network of Central London. The probability distribution for roads $\operatorname{Pr}\left(n_{r}\right)$ (14a) appears highly plausible, as expected for any recognized self-organized city (Alexander 1965; Jiang et al. 2014). However, for the time being, the validation of the probability distribution for junctions $\operatorname{Pr}\left(n_{j}\right)(14 \mathrm{~b})$ appears more delicate. This is due to the emergence of a numerical bottleneck as follows. The state-of-the-art statistical method to either validate or reject a plausible hypothesis for power law probability distributions is based on Maximum Likelihood Estimations (MLE) (Clauset et al. 2009). Besides invoking a numerical minimizer (Press et al. 2007), this method requires sampling (Clauset et al. 2009), that is, the input sample must be compared to a large set of randomly generated samples - the larger, the more precise. In the present case, this means that the numerical evaluation of the normalizing functions $\zeta$ and $\mathcal{W}$ - and of their respective logarithms and logarithmic derivatives - have to be efficient not only in terms of precision but also in terms of speed. Efficient 


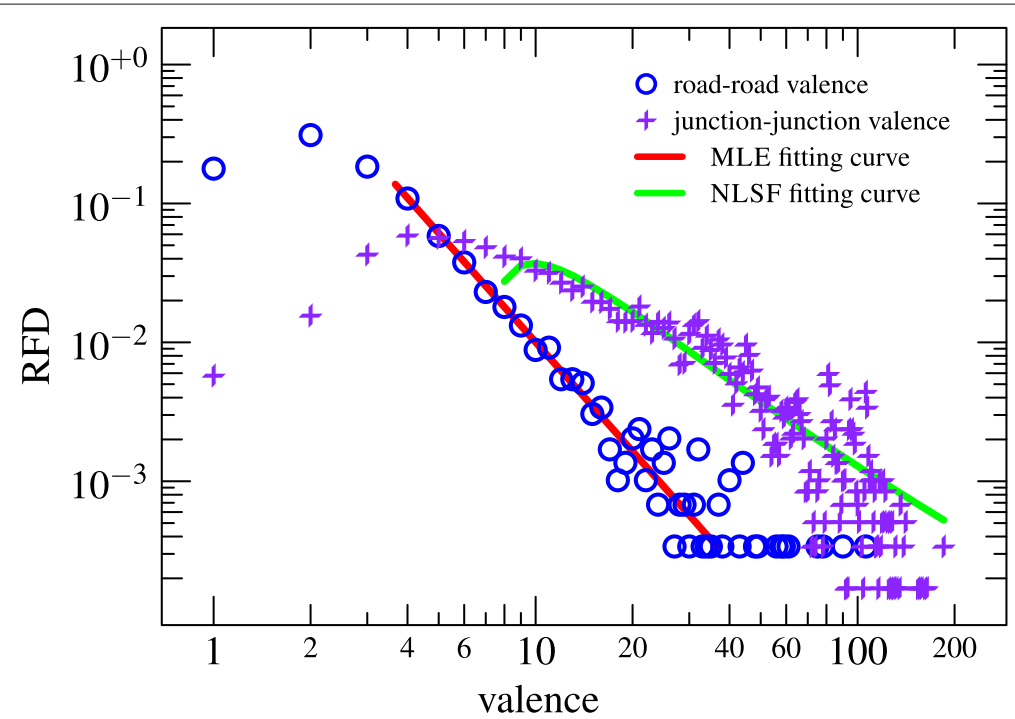

Fig. 4 Relative Frequency Distributions (RFD) for the urban street network of Central London: circles represent relative frequencies for the valences of the road-road topological network; crosses represent relative frequencies for the valences of the junction-junction topological network. The red fitted curve for the natural road statistics describes the Maximum Likelihood Estimate (MLE) for the discrete Pareto probability distribution (14a) estimated according to the state of the art (Clauset et al. 2009; Gillespie 2015) $\underline{n}_{r}=4$, $2 \lambda v_{r}=2.610(65), n=250000$ samples, $p$-value $\left.=0.933(1)\right)$. The green fitted curve for the junction statistics shows the best Nonlinear Least-Squares Fitting (NLSF) for the nonstandard discrete probability distribution (14b) with $\underline{n}_{r}$ and $2 \lambda v_{r}$ fixed to their respective MLE value $\left(2 \lambda v_{j} \approx-1.3\right)$; since fast evaluation of the normalizing function $\mathcal{W}$ has yet to be found, no MLE approach can be used for now. Having for junctions a number of vital connections $v_{j}$ negative is interpreted as expressing a number of agent intraconnections for junctions relatively much smaller than the one for natural roads. The sharp downturn at a valence of 10 likely means that the model fails to catch what occurs when valences are small. In any case, a proper MLE remains to be performed for confirming

numerical methods to evaluate the Hurwitz-zeta function $\zeta$ can be found in the classical numerical literature (Olver et al. 2010; Oldham et al. 2009) - while they can easily be adapted to our specific usage. By contrast, the two-dimensional Mordell-TornheimWitten zeta function $\mathcal{W}$ belongs to the specialized numerical literature and its numerical computation is still a subject of investigation (Borwein and Dilcher 2018). In practice, even the implementation of the corresponding Hurwitz generalization with the same two first exponents $\alpha$ and $\beta$ is rather tedious while very slow, especially when the third exponent $\gamma$ becomes negative - as $2 \lambda v_{j}$ appeared to be. To work around this numerical bottleneck, we performed a crude data analysis based on a Nonlinear Least-Squares Fitting (NLSF). Interestingly, our ad hoc crude data analysis reveals a negative number of vital connection $v_{j}$, which means that the associated generalized binomial combination number is smaller than one modulo a signed factor that drops at normalization ${ }^{2}$. We interpret this to mean that the number of intraconnections for junctions might be relatively much smaller than the one for roads in self-organized cities.

\section{Drifted self-organized urban street networks}

\section{Coherence based on drifted Boltzmannian mesoscopic surprisals}

Now let us regard the self-organized urban street networks studied in the previous section as an ideal class of urban street networks, namely, as a reference from which 'real' urban 
street networks deviate. The deviation is vanishing for self-organized urban street networks. For arbitrary urban street networks, the deviation might be of arbitrary magnitude. Furthermore, we presume that deviations are essentially caused by artificial means, but not due to any change in the behaviours of the city-dwellers. Artificial deviations are created by urban designers or decision makers who remodel cities for arbitrary purposes but without respect to the laws that might govern the spontaneous evolution of cities. Meanwhile, the topological mindset of city-dwellers and the social machinery that governs roads and junctions remain unchanged. Moreover, a priori, there are no apparent reason that the remodelling affects one iota the deep paradigm which constructs the perception of city-dwellers: roads and junctions remain perceived as Boltzmannian mesoscopic systems. Nevertheless, the remodeled urban street networks might no more reflect their perception - not vice versa. In other words, the deviations drift the surprisal of city-dwellers for their own urban street network. Assuming a surprisal drift $\Lambda(\Omega)$ that generates an extra amount of surprisal $\Delta\langle\mathrm{S}\rangle$ on average, the unique characterizing constraint bracket in Shannon Lagrangian (8) becomes

$$
\left[\sum_{\Omega} \operatorname{Pr}(\Omega)(\ln \Omega+\Lambda(\Omega))-(\langle\mathrm{S}\rangle+\Delta\langle\mathrm{S}\rangle)\right] .
$$

Carefully expanding (15) gives rise to two apparent characterizing restrictions: the first logarithmic moment characterizing constraint discussed above and a new characterizing constraint, respectively

$$
\left[\sum_{\Omega} \operatorname{Pr}(\Omega) \ln (\Omega)-\langle\mathrm{S}\rangle\right] \text { and }\left[\sum_{\Omega} \operatorname{Pr}(\Omega) \Lambda(\Omega)-\Delta\langle\mathrm{S}\rangle\right] .
$$

By adding this new characterizing restriction to Shannon Lagrangian (8), we arrive at the deviant version

$$
\begin{gathered}
\mathcal{L}(\{\operatorname{Pr}(\Omega)\} ; \nu, \lambda, \varepsilon)=-\sum_{\Omega} \operatorname{Pr}(\Omega) \ln (\operatorname{Pr}(\Omega))-(v-1)\left[\sum_{\Omega} \operatorname{Pr}(\Omega)-1\right] \\
-\lambda\left[\sum_{\Omega} \operatorname{Pr}(\Omega) \ln \Omega-\langle\mathrm{S}\rangle\right]-\varepsilon\left[\sum_{\Omega} \operatorname{Pr}(\Omega) \Lambda(\Omega)-\Delta\langle\mathrm{S}\rangle\right] .
\end{gathered}
$$

The introduced Lagrange multiplier $\varepsilon$ tells us how urban designers or decision makers impose a surprisal drift $\Lambda(\Omega)$ to the surprisal perception of city-dwellers for their own urban street network. The constant $\Delta\langle\mathrm{S}\rangle$ corresponds to the part of the apparent mean surprisal caused by the surprisal drift $\Lambda(\Omega)$ itself - for now, as the constant $\langle\mathrm{S}\rangle$, it plays a dummy role. Extremizing expression (17) holds

$$
\frac{\partial \mathcal{L}(\{\operatorname{Pr}(\Omega)\} ; \nu, \lambda, \varepsilon)}{\partial \operatorname{Pr}(\Omega)}=-\ln (\operatorname{Pr}(\Omega))-v-\lambda \ln \Omega-\varepsilon \Lambda(\Omega)=0,
$$

from which we readily find the power law probability distribution

$$
\operatorname{Pr}(\Omega)=\frac{\Omega^{-\lambda} e^{-\Lambda(\Omega)}}{e^{\nu}} .
$$

With the same easy manipulation as before, the normalization condition allows us to define the deviant partition function $Z(\Lambda ; \lambda, \varepsilon)$ of our drifted system; we get

$$
e^{\nu}=\sum_{\Omega} \Omega^{-\lambda} e^{-\varepsilon \Lambda(\Omega)} \equiv Z(\Lambda ; \lambda, \varepsilon) .
$$


So we end up by writing the most plausible probability distribution associated to Shannon Lagrangian (17) as

$$
\operatorname{Pr}(\Omega)=\frac{\Omega^{-\lambda} e^{-\varepsilon \Lambda(\Omega)}}{Z(\Lambda ; \lambda, \varepsilon)} .
$$

For non-vanishing surprisal drift $\varepsilon \Lambda(\Omega)$, as expected, this probability distribution is obviously not scale-free. In fact, when the polynomial part of the asymptotic expansion of $\Lambda(\Omega)$ does not reduce to a constant, the surprisal drift $\varepsilon \Lambda(\Omega)$ acts as a cut-off function. In other words, in contrast to ideal self-organized urban street networks, a typical deviant urban street network possesses a typical number of configurations for its roads and junctions.

Our next task is to establish the statistics for roads and junctions in deviant urban street networks. Substitution of (7a) into (12) yields

$$
\operatorname{Pr}\left(n_{r}\right) \propto n_{r}^{-2 \lambda v_{\mathrm{r}}} \exp \left(-\varepsilon \tilde{\Lambda}_{\mathrm{r}}\left(n_{r}^{2 v_{\mathrm{r}}}\right)\right),
$$

once the surprisal drift $\Lambda$ is suitably rescaled to the surprisal drift for roads $\widetilde{\Lambda}_{\mathrm{r}}$. Afterwards, substitution of (7b) into (12) along Iversonian counting with respect to (22a) gives

$$
\begin{aligned}
\operatorname{Pr}\left(n_{j}\right) \propto\left(\sum_{j(r, s)} \frac{\left[n_{j}=n_{r}+n_{s}\right]}{\left(n_{r} n_{s}\right)^{2 \lambda v_{\mathrm{r}}}} \exp \left(-\varepsilon\left[\widetilde{\Lambda}_{\mathrm{r}}\left(n_{r}^{2 v_{\mathrm{r}}}\right)+\widetilde{\Lambda}_{\mathrm{r}}\left(n_{s}^{2 v_{\mathrm{r}}}\right)\right]\right)\right) \\
\times n_{j}^{-2 \lambda v_{\mathrm{j}}} \exp \left(-\varepsilon \widetilde{\Lambda}_{\mathrm{j}}\left(n_{j}^{2 v_{\mathrm{j}}}\right)\right),
\end{aligned}
$$

with the same notation convention previously used.

The main interest of the deviant statistics (22) lies in showing how surprisal drift formally separates the evolution scale exponent $\lambda$ from the numbers of vital connections for roads and junctions, $v_{\mathrm{r}}$ and $v_{\mathrm{j}}$ respectively. As seen in the previous subsection, this separation of parameters is important as it means that the macroscopic phenomenon of evolution and the mesoscopic phenomena of social interactions can be qualitatively studied among drifted self-organized urban street networks. Fortunately enough, such qualitative investigations among slightly drifted self-organized urban street networks appears almost as manageable as the ideal case investigation among self-organized urban street networks as follows.

\section{Exploratory study of slightly drifted urban street networks}

Let us first specify what we mean when a self-organized urban street network is slightly drifted. Here it is important to bear in mind that the numbers of configurations (7) result from asymptotic countings. So, the surprisal drifts for roads and junctions introduced in (22), $\widetilde{\Lambda}_{\mathrm{r}}$ and $\tilde{\Lambda}_{j}$ respectively, reach the asymptotic behaviour of the underlying surprisal drift $\Lambda$. Let us now assume that the underlying surprisal drift $\Lambda(\Omega)$ admits as asymptotic expansion a generic finite Laurent polynomial of the form $a_{-p} \Omega^{-p}+a_{-p+1} \Omega^{-p+1}+\cdots+$ $a_{0}+\cdots+a_{q-1} \Omega^{q-1}+a_{q} \Omega^{q}$. The non-polynomial part is absorbed by the exponential function whose the surprisal drifts feed, hence irrelevant. The zeroth order coefficient $a_{0}$ is eliminated during the normalization by factorizing its inverse exponentiation, hence meaningless. The remaining polynomial part $a_{1} \Omega+\cdots+a_{q-1} \Omega^{q-1}+a_{q} \Omega^{q}$ is imposed, by the normalization condition, to be positive for large $\Omega$ values. More importantly, the remaining polynomial behaves as an asymptotic cut-off polynomial whose strength lies 
in its leading term $a_{q} \Omega^{q}$. We may consider surprisal drifts asymptotic to quadratic or of higher degree polynomials as inducing too drastic cut-offs, namely, as altering too drastically self-organized urban street networks. That is, for the time being, we consider as slight any surprisal drift that is asymptotic to a monomial of degree one $a_{1} \Omega$ whose coefficient $a_{1}$ is arbitrary small - and positive. Thus, we may assume, without loss of generality, that the slight surprisal drift $\Lambda(\Omega)$ reduces to the canonical monomial $\Omega$ so that $\varepsilon \Lambda(\Omega)=\varepsilon \Omega$. Therefore the parameter $\varepsilon$ simply expresses the strength of our slight surprisal drift. Once properly rescaled, the parameter $\varepsilon$ becomes the strengths $\widetilde{\varepsilon}_{\mathrm{r}}$ and $\widetilde{\varepsilon}_{\mathrm{j}}$ associated to the slight surprisal drifts for roads and junctions, respectively; we write

$$
\begin{aligned}
\varepsilon \widetilde{\Lambda}_{\mathrm{r}}\left(n_{r}^{2 v_{\mathrm{r}}}\right) & =\widetilde{\varepsilon}_{\mathrm{r}} n_{r}^{2 v_{\mathrm{r}}} \\
\varepsilon \widetilde{\Lambda}_{\mathrm{j}}\left(n_{j}^{2 v_{\mathrm{j}}}\right) & =\widetilde{\varepsilon}_{\mathrm{j}} n_{j}^{2 v_{\mathrm{j}}} .
\end{aligned}
$$

Now we may carry out the statistics for roads and junctions in slightly deviant urban street networks. Substituting (23) into (22), then making the change of parameters

$$
\mathfrak{z}_{\mathrm{r}}=\exp \left(-\widetilde{\varepsilon}_{\mathrm{r}}\right) \quad \mathfrak{z}_{\mathrm{j}}=\exp \left(-\widetilde{\mathcal{\varepsilon}}_{\mathrm{j}}\right)
$$

for conciseness, we obtain

$$
\begin{aligned}
& \operatorname{Pr}\left(n_{r}\right) \propto n_{r}^{-2 \lambda v_{\mathrm{r}}} \mathfrak{z}_{\mathrm{r}}^{n_{r}^{2 v_{\mathrm{r}}}} \\
& \operatorname{Pr}\left(n_{j}\right) \propto\left(\sum_{j(r, s)} \frac{\left[n_{j}=n_{r}+n_{s}\right]}{\left(n_{r} n_{s}\right)^{2 \lambda v_{\mathrm{r}}}} \mathfrak{z}_{\mathrm{r}}^{n_{r}^{2 v_{\mathrm{r}}}} \mathfrak{j}_{\mathrm{r}}^{n_{s}^{2 v_{\mathrm{r}}}}\right) n_{j}{ }^{-2 \lambda v_{\mathrm{j}}} \mathfrak{j}_{j}^{n_{j}^{2 v_{j}} .} .
\end{aligned}
$$

For practical normalization, the change of parameters (24) appears precious to easily identify the involved special functions. First, the probability (14a) for a road to cross $n_{r}$ junctions in an idealized self-organized urban street network takes in a slightly deviant urban street network the form

$$
\operatorname{Pr}\left(n_{r}\right)=\frac{n_{r}^{-2 \lambda v_{\mathrm{r}}} \mathfrak{z r}^{n_{r}^{2 v_{\mathrm{r}}}}}{\Phi\left(\mathfrak{z}_{\mathrm{r}}, 2 \lambda v_{r}, \underline{n}_{r} ; 2 v_{\mathrm{r}}\right)},
$$

where $\Phi(z, \alpha, a ; \beta)=\sum_{n=0}^{\infty}(a+n)^{-\alpha} z^{(a+n)^{\beta}}$ is the generalization introduced by Johnson (1974) of the Lerch transcendent function $\Phi(z, \alpha, a)=\sum_{n=0}^{\infty}(a+n)^{-\alpha} z^{n}$ ((Olver et al. 2010), $\mathbb{2} 25.14$ ). Second, the concomitant probability (14b) for a junction to see $n_{j}$ junctions through its joining roads then transforms into

$$
\operatorname{Pr}\left(n_{j}\right)=\frac{\sum_{n=\underline{\underline{n}}_{r}}^{n_{j}-\underline{\underline{n}}_{r}}\left[n\left(n_{j}-n\right)\right]^{-2 \lambda v_{r}} \mathfrak{z}_{\mathrm{r}}{ }^{2 v_{\mathrm{r}}} \mathfrak{z}_{\mathrm{r}}\left(n_{j}-n\right)^{2 v_{\mathrm{r}}} n_{j}^{-2 \lambda v_{j}} \mathfrak{z}_{j}{ }^{2 v_{j}}}{\mathcal{W}_{\Phi}\left(\left[\mathfrak{z}_{\mathrm{r}}, \mathfrak{z}_{\mathrm{r}}, \mathfrak{z}_{\mathfrak{j}}\right],\left[2 \lambda v_{r}, 2 \lambda v_{r}, 2 \lambda v_{j}\right] ; \underline{n}_{r} ;\left[2 v_{r}, 2 v_{r}, 2 v_{j}\right]\right)},
$$

where

$$
\mathcal{W}_{\Phi}([x, y, z],[\alpha, \beta, \gamma] ; \underline{;} ;[\iota, \kappa, \mu])=\sum_{m, n \geqslant \underline{n}} m^{-\alpha} n^{-\beta}(m+n)^{-\gamma} x^{m^{\iota}} y^{n^{\kappa}} z^{(m+n)^{\mu}}
$$

is introduced for the sake of completeness.

The validation of the slightly deviant statistics (26) is more challenging than of the ideal statistics (14) from which it deviates for at least two reasons. Firstly, it is rather an exploratory work since we have no catalogue of slightly deviant urban street networks from which we can pick up relevant samples. Secondly, the involved normalizing functions $\Phi$ and $\mathcal{W}_{\Phi}$ are both computationally challenging. Nonetheless, the numerical bottleneck is again a bearer of both good news and bad news. 
The bad news, without surprise, is that further investigations on the deviant probability distribution $\operatorname{Pr}\left(n_{j}\right)$ (26b) for junctions must be postponed too. This is simply because its normalizing function $\mathcal{W}_{\Phi}$ combines together the difficulties inherited from the normalizing functions $\mathcal{W}$ and $\Phi$ while, for the least, a fast numerical evaluation for the former has yet to be found. The good news is that a very efficient numerical evaluation already exists for the latter. In fact, this numerical evaluation was presented with the Lerch transcendent function as illustration (Aksenov et al. 2003). Formally, it consists to apply to the series a condensation transformation followed by a Levin d-transformation (Press et al. 2007; Brezinski and Redivo Zaglia 1991). Technically, its adaptation to the generalized Johnson-Lerch transcendent function $\Phi$ is straightforward. In practice, a careful implementation written in $C$ language that uses the Levin transformation encoded in the HURRY procedure (Fessler et al. (1983), Algo. 602) as implemented in the GNU Scientific Library (Galassi et al. 2009) appears efficient in terms of both precision and speed.

\section{Conclusions and future works}

The primary goal of our investigation is to understand the statistics of urban street networks. The objective of this research was two-fold. First, see how our recent results on self-organized urban street networks can be broaden to 'nearly' self-organized urban street networks. Second, learn what we can expect from this extension of our initial domain of investigation. The implicit idea behind this approach is that most urban street networks can be envisaged as a perturbation of a self-organized urban street network.

To start, we present the surprisal statistical physics model that we showed to govern self-organized urban street networks. Afterwards, by hand, we perturb the model by introducing a surprisal drift. We argue that the surprisal drift essentially results from artificial remodellings imposed by urban designers or decision makers. We obtain the generic statistics for arbitrarily drifted self-organized urban street networks, and most importantly, a practical statistics for the slightly drifted ones among them. All along we learn two important and practical properties. First, as expected whenever any perturbation occurs, surprisal drift perturbations lead to a separation of parameters. Here, perturbations separate the macroscopic evolution scale parameter from the mesoscopic social interaction parameters. Second, data analysis for validating the practical statistics for slightly drifted self-organized urban street networks remains manageable - modulo some numerical analysis efforts.

Future works must first and foremost validate the practical statistic for slightly drifted self-organized urban street networks for a sufficiently large bunch of 'real' urban street networks. Thereafter, the macroscopic and mesoscopic parameters must be estimated for a representative set of slightly drifted self-organized urban street networks in view to perform an observational qualitative investigation on the involved phenomena. This investigation is helpful to determine whether or not the macroscopic phenomena of evolution and the mesoscopic phenomena of social interactions transcend cultural basins. There exists evidences that the latters are cultural dependent. We believe, in contrast, that the macroscopic phenomena of evolution is characterized by an universal constant evolution scale that might reflect either spacial spanning, unconscious processes, or both; if so, an observational estimation must be isolated and ultimately some rational must be found. This sequence of observational investigations aims to confirm for 
self-organized urban street networks the status of reference among urban street networks. Once confirmed, it makes sense to compare the strengths of the surprisal drifts among a representative set of urban street networks. Because these strengths reflect the rational thoughts of urban designers and decision makers, we expect to observe a random (not to say irrational) set of data. On the other hand, since surprisal drifts might be perceived as a source of stress by city-dwellers, these strengths might have correlation with data that mark their disengagement for their own urban street networks. If such correlations are effectively observed, then the strength of surprisal drifts might be interpreted as a indicator of their disengagement, namely, as a quality measure of their city.

\section{Endnotes}

${ }^{1}$ We have $\left(\begin{array}{c}N \\ -v\end{array}\right)=\frac{\sin \pi v}{\pi v}\left(\begin{array}{c}N+v \\ v\end{array}\right)^{-1}=\frac{\sin \pi v}{\pi v}\left(\begin{array}{c}N \\ v\end{array}\right){ }^{-1}\left(1+\mathcal{O}\left(\frac{v^{2}}{N}\right)\right)$.

${ }^{2}$ Notional example inspired by the 'notional road network' in the paper by Jiang et al. (2008).

\section{Abbreviations}

FCA: Formal concept analysis; MLE: Maximum likelihood estimate; NLSF: Nonlinear least-squares fitting; RFD: Relative frequency distribution

\section{Authors' contributions}

JGMB conceived and designed the study, programmed the map treatment/analysis tools, collected and treated the map data, performed the statistical analysis, and wrote the manuscript. SEGJ helped to shape the manuscript. Both authors read and approved the final manuscript.

\section{Availability of data and materials}

The datasets generated and analysed during the current study are available from the corresponding author on reasonable request.

\section{Competing interests}

The authors declare that they have no competing interests.

\section{Author details}

${ }^{1}$ New York University Abu Dhabi, Saadiyat Island, POB 129188, Abu Dhabi, UAE. ${ }^{2}$ New York University

Tandon School of Engineering, Brooklyn, NY 11201, New York, USA.

Received: 15 March 2019 Accepted: 6 June 2019

Published online: 16 July 2019

\section{References}

Aczél J, Forte B, Ng CT (1974) Why the Shannon and Hartley entropies are 'natural'. Adv Appl Probab 6(1):131-146. https://doi.org/10.2307/1426210

Aksenov SV, Savageau MA, Jentschura UD, Becher J, Soff G, Mohr PJ (2003) Application of the combined nonlinear-condensation transformation to problems in statistical analysis and theoretical physics. Comput Phys Comm 150(1):1-20. https://doi.org/10.1016/S0010-4655(02)00627-6

Alexander C (1965) A city is not a tree. Arch Forum 122(1+2):58-62

Atkin RH (1974) Mathematical Structure in Human Affairs. Heinemann Educational Books, London

Benoit J, Jabari SE (2019) Structure entropy, self-organization and power laws in urban street networks. https://arxiv.org/ abs/1902.07663

Borwein JM, Dilcher K (2018) Derivatives and fast evaluation of the Tornheim zeta function. Ramanujan J 45(2):413-432. https://doi.org/10.1007/s11139-017-9890-9

Brezinski C, Redivo Zaglia M (1991) Extrapolation Methods: Theory and Practice, Studies in Computational Mathematics, vol 2. 1st edn. Elsevier Science B. V., Amsterdam

Clauset A, Shalizi CR, Newman MEJ (2009) Power-law distributions in empirical data. SIAM Rev 51(4):661-703. https://doi. org/10.1137/070710111

Crucitti P, Latora V, Porta S (2006) Centrality measures in spatial networks of urban streets. Phys Rev E 73(3):036,125. https://doi.org/10.1103/PhysRevE.73.036125

Davey BA, Priestley HA (2002) Introduction to Lattices and Order. 2nd edn. Cambridge University Press, Cambridge. https://doi.org/10.1017/CBO9780511809088

Dover $Y$ (2004) A short account of a connection of power laws to the information entropy. Physica A 334(3-4):591-599. https://doi.org/10.1016/j.physa.2003.09.029

Dunbar RIM, Shultz S (2007) Evolution in the social brain. Science 317(5843):1344-1347. https://doi.org/10.1126/science. 1145463 
Fessler T, Ford WF, Smith DA (1983) HURRY: An acceleration algorithm for scalar sequences and series. ACM Trans Math Softw 9(3):346-354. https://doi.org/10.1145/356044.356051

Galassi M, Davies J, Theiler J, Gough B, Jungman G, Alken P, Booth M, Rossi F (2009) GNU Scientific Library Reference Manual. 3rd edn. Network Theory Limited. https://www.worldcat.org/isbn/9780954612078

Gillespie CS (2015) Fitting heavy tailed distributions: The poweRlaw package. J Stat Softw 64(2):1-16. https://doi.org/10. 18637/jss.v064.i02

Graham RL, Knuth DE, Patashnik O (1994) Concrete Mathematics: A Foundation for Computer Science. 2nd edn. Addison-Wesley, Reading

Ho YS (1982) The planning process: Structure of verbal descriptions. Environ Plan B 9(4):397-420. https://doi.org/10.1068/ b090397

Jaynes ET (1957a) Information theory and statistical mechanics. Phys Rev 106(4):620-630. https://doi.org/10.1103/ PhysRev.106.620

Jaynes ET (1957b) Information theory and statistical mechanics ii. Phys Rev 108(2):171-190. https://doi.org/10.1103/ PhysRev. 108.171

Jaynes ET (1989) Where Do We Stand on Maximum Entropy?, Synthese Library, vol 158. Kluwer Academic Publishers, Dordrecht. https://doi.org/10.1007/978-94-009-6581-2

Jiang B (2007) A topological pattern of urban street networks: Universality and peculiarity. Physica A 384(2):647-655. https://doi.org/10.1016/j.physa.2007.05.064

Jiang B, Claramunt C (2004) Topological analysis of urban street networks. Environ Plan B 31(1):151-162. https://doi.org/ $10.1068 / \mathrm{b} 306$

Jiang B, Liu C (2009) Street-based topological representations and analyses for predicting traffic flow in GIS. Int J Geogr Inf Sci 23(9):1119-1137. https://doi.org/10.1080/13658810701690448

Jiang B, Zhao S, Yin J (2008) Self-organized natural roads for predicting traffic flow: A sensitivity study. J Stat Mech Theor Exp 2008(7):P07008. https://doi.org/10.1088/1742-5468/2008/07/P07008

Jiang B, Duan Y, Lu F, Yang T, Zhao J (2014) Topological structure of urban street networks from the perspective of degree correlations. Environ Plan B 41(5):813-828. https://doi.org/10.1068/b39110

Johnson BR (1974) Generalized Lerch zeta function. Pacific J Math 53(1):189-193. https://doi.org/10.2140/pjm.1974.53.189

Kapur JN, Kesavan HK (1992) Entropy Optimization Principles and Their Applications, Water Science and Technology Library, vol 9. Springer-Verlag, Dordrecht. https://doi.org/10.1007/978-94-011-2430-0

Kesavan HK (2009) Jaynes' Maximum Entropy Principle. 2nd edn. Springer-Verlag, Boston

Knuth DE (1992) Two notes on notation. Amer Math Monthly 99(5):403-422. https://doi.org/10.2307/2325085

Knuth KH (2005) Lattice duality: The origin of probability and entropy. Neurocomputing 67:245-274. https://doi.org/10. 1016/j.neucom.2004.11.039

Knuth KH (2008) The origin of probability and entropy. AIP Conf Proc 1073(1):35-48. https://doi.org/10.1063/1.3039020 Knuth KH (2009) Measuring on lattices. AIP Conf Proc 1193(1):132-144. https://doi.org/10.1063/1.3275606

Knuth KH (2011) Information physics: The new frontier. AIP Conf Proc 1305(1):3-19. https://doi.org/10.1063/1.3573644

Knuth KH (2014) Information-based physics: An observer-centric foundation. Contemp Phys 55(1):12-32. https://doi.org/ 10.1080/00107514.2013.853426

Masucci AP, Molinero C (2016) Robustness and closeness centrality for self-organized and planned cities. Eur Phys J B 89:53. https://doi.org/10.1140/epjb/e2016-60431-2

Masucci AP, Smith D, Crooks A, Batty M (2009) Random planar graphs and the London street network. Eur Phys J B 71:259-271. https://doi.org/10.1140/epjb/e2009-00290-4

Milaković M (2001) A statistical equilibrium model of wealth distribution. Computing in Economics and Finance 2001 214, Society for Computational Economics. https://ideas.repec.org/p/sce/scecf1/214.html

Oldham KB, Myland JC, Spanier J (2009) An Atlas of Functions: with Equator, the Atlas Function Calculator. 2nd edn. Springer-Verlag, New York. https://doi.org/10.1007/978-0-387-48807-3

Olver FWJ, Lozier DW, Boisvert RF, Clark CW (2010) NIST Hand Book of Mathematical Functions. Cambridge University Press. https://dlmf.nist.gov/

Porta S, Crucitti P, Latora V (2006a) The network analysis of urban streets: A dual approach. Physica A 369(2):853-866. https://doi.org/10.1016/j.physa.2005.12.063

Porta S, Crucitti P, Latora V (2006b) The network analysis of urban streets: A primal approach. Environ Plan B 33(5):705-725. https://doi.org/10.1068/b32045

Press WH, Teukolsky SA, Vetterling WT, Flannery BP (2007) Numerical Recipes: The Art of Scientific Computing. 3rd edn. Cambridge University Press, Cambridge

Rosvall M, Trusina A, Minnhagen P, Sneppen K (2005) Networks and cities: An information perspective. Phys Rev Lett 94(2):028701. https://doi.org/10.1103/PhysRevLett.94.028701

Rota GC (1971) On the Combinatories of the Euler Characteristic, London Mathematical Society Monographs, vol $1: 221-233$

Tribus M (1961) Thermostatics and Thermodynamics. Van Nostrand, Princeton. https://hdl.handle.net/2027/mdp. 39015001333361

West GB (2017) Scale: the Universal Laws of Growth, Innovation, Sustainability, and the Pace of Life in Organisms, Cities, Economies, and Companies. Penguin Press, New York

\section{Publisher's Note}

Springer Nature remains neutral with regard to jurisdictional claims in published maps and institutional affiliations. 